12

\title{
Гомодинный квадратурный интерферометр перемещений. Моделирование
}

\author{
(C) Г.Н. Вишняков ${ }^{1,2}$, В.Л. Минаев ${ }^{1,3}$ \\ ${ }^{1}$ Всероссийский научно-исследовательский институт оптико-физических измерений (ФГУП „ВНИИОФИ“), \\ 119361 Москва, Россия \\ ${ }^{2}$ МГТУ имени Н.Э. Баумана, \\ 105005 Москва, Россия \\ ${ }^{3}$ НИу „Высшая школа экономики“, \\ 101000 Москва, Россия \\ e-mail:vish@vniiofi.ru
}

Поступила в редакцию 08.06.2021 г.

В окончательной редакции 08.06.2021 г.

Принята к публикации 24.06.2021 г.

На основе формализма матриц Джонса описана оптическая схема однопроходного гомодинного интерферометра перемещений с квадратурным принципом регистрации фазы. Интерферометр построен по схеме Майкельсона, а для получения квадратурных сигналов используются поляризационные оптические элементы. Интерферометр предполагается использовать в составе нового отечественного эталона килограмма на основе ватт-весов для прецизионных измерений перемещений и скорости катушки в вертикальном направлении. Рассмотрены результаты моделирования оптической схемы интерферометра с целью оценки влияния на точность измерений несовершенства поляризационных элементов и качества их юстировки. Также реализован алгоритм компенсации нелинейных эффектов, возникающих при квадратичном детектировании интерференционных сигналов.

Ключевые слова: интерферометр перемещений, гомодинный, квадратурный сигнал, ватт-весы.

DOI: $10.21883 /$ OS.2021.10.51498.2400-21

\section{Введение}

Интерферометры перемещений имеют широкий спектр приложений, включая позиционирование столика станка и контроль расстояния по шкалам длины от нескольких миллиметров до сотен километров в космических системах [1]. Длина волны света обеспечивает очень точное измерение расстояний и является основой для коммерческих интерферометрических измерительных инструментов, которые отслеживают положение объектов с разрешением лучше $1 \mathrm{~nm}$. В дополнение к высокому разрешению, широкому диапазону измерения и быстрому отклику лазерный луч представляет собой виртуальную ось измерения, которая может проходить непосредственно через интересующую точку измерения для устранения ошибок смещения Аббе $[1,2]$. Поэтому интерферометры перемещений используются и в первичных национальных эталонах, например, в эталоне единицы длины ГЭТ 2-2021. В новом эталоне единицы массы килограмма на основе ватт-весов также используются интерферометры перемещений для измерения перемещений и скорости катушки и пробной массы в вертикальном направлении.

Как известно, лазерные интерферометры перемещений бывают двух типов - гомодинные и гетеродинные [1]. Ключевым отличием между гомодинным и гетеродинным интерферометрами является частота, на которой происходит измерение. В гетеродинном интер- ферометре спектр полезного сигнала переносится на частоту модуляции длины волны лазерного излучения (внешняя модуляция), либо оптической длины пути опорного канала (внутренняя фазовая модуляция) [2].

Достоинства и недостатки гомодинных и гетеродинных интерферометров рассматривались во многих работах, например, в обзоре [3]. Гомодинные интерферометры обладают компактностью, простотой оптической схемы, а также меньшей нелинейностью при измерении перемещений [4]. Для восстановления разности фаз между интерферирующими пучками, как правило, используются квадратурные сигналы, а для их получения применяют различные оптические схемы с поляризационными элементами.

Для достижения субнанометровой точности измерений перемещений необходимо учитывать и компенсировать нелинейность интерферометров, которая возникает от несовершенства поляризационных элементов, в первую очередь поляризационного светоделителя, различием в чувствительности и коэффициентах усиления детекторов ит. П. [4]. Поэтому в настоящее время активно ведутся работы по компенсации нелинейности интерферометров как с помощью численных методов в первую очередь с помощью широко распространенного итерационного алгоритма вычисления параметров эллипса (elliptical least-squares fitting technique) [5], так и аппаратными методами [6-8]. 
В настоящей работе выбрана оптическая схема гомодинного интерферометра перемещений с квадратурным принципом регистрации фазы, который предполагается использовать в составе нового отечественного эталона килограмма. Цель работы состоит в математическом моделировании оптических полей, которые возникают после прохождения поляризационных элементов, что позволило оценить влияние на измерения перемещений неточности установки поляризационных элементов и качества их юстировки. Также рассмотрен и промоделирован алгоритм компенсации нелинейных эффектов, возникающих при квадратичном детектировании интерференционных сигналов. При моделировании использовался формализм матриц Джонса, с помощью которого в работе получены формулы для квадратурных сигналов.

\section{Оптическая схема гомодинного интерферометра}

В настоящей работе выбрана оптическая схема однопроходного гомодинного интерферометра перемещений с квадратурным принципом регистрации фазы, аналогичная той, которая используется в составе ватт-весов национального метрологического института Южной Кореи „KRISS“ [9]. Чертеж макета гомодинного квадратурного интерферометра перемещений приведен на рис. 1. Макет полностью спроектирован из покупных изделий фирмы ThorLabs, а для крепления и юстировки оптико-механических элементов используется кейдж-система этой же фирмы.

В качестве источника излучения используется $\mathrm{He}-\mathrm{Ne}$-лазер, стабилизированный по частоте, с волоконным выходом с сохранением поляризации и коллиматором 1. Направление плоскости поляризации линейно поляризованного лазерного излучения должно составлять $45^{\circ}$ к горизонтальной плоскости оптического стола ( $x$-плоскости, совпадающей с плоскостью листа). Доставленное излучение после отражения от первой зеркальной грани призмы 2 поступает в интерферометр Майкельсона, состоящий из поляризационного светоделительного кубика 3, неподвижного 4 и подвижного 5 трехгранных ретроотражателей (триппель-призм). После поляризационного светоделительного кубика 3 формируются два пучка света с ортогональной поляризацией, которые после отражения от триппель-призм 4, 5 и второй зеркальной грани призмы 2 поступают в регистрирующий блок интерферометра.

Четвертьволновая пластинка 6 , быстрая ось которой расположена под углом $45^{\circ}$ к горизонтальной плоскости, преобразует поляризацию этих лучей в круговую с противоположным направлением вращения векторов напряженности электрического поля световой волны. Далее эти пучки после отражения и прохождения через неполяризационный 7 и поляризационный 10 светоделительные кубики направляются к волоконным коллиматорам 9, 11,
12, которые соединены волоконными жгутами с соответствующими фотодетекторами (на рис. 1 не показаны). При этом излучение, которое направляется к коллиматору 9, вначале проходит линейный поляризатор 8, азимут которого составляет угол $45^{\circ}$ с горизонтальной плоскостью.

Смещение ретроотражателя 5 в предметном плече интерферометра измеряется путём детектирования фазы сигнала, образующегося в результате интерференции лучей. В общем случае для вычисления фазы сигнала необходимы две квадратурных компоненты, однако в данной установке используется три - $0^{\circ}, 90^{\circ}$ и $180^{\circ}$. Это необходимо для частичного устранения постоянной составляющей сигналов.

\section{Применение формализма Джонса для описания оптической схемы гомодинного интерферометра}

Рассмотрим подробнее, как образуются квадратурные сигналы с помощью поляризационных элементов. Для этого используем формализм матриц Джонса [10]. Лазерное излучение, попадающее в интерферометр из волоконного коллиматора 1 , после поляризационного светоделительного кубика 3 (рис. 1) делится на два пучка с ортогональной линейной поляризацией. Причем отраженное от светоделительной грани кубика излучение будет иметь вертикальную линейную поляризацию (вдоль оси $y$ ), а прошедшее излучение - горизонтальную линейную поляризацию (вдоль оси $x$ ). Для горизонтальной и вертикальной поляризаций нормированные векторы Джонса записываются в следующем виде[10]:

$$
\begin{gathered}
\mathbf{E}(0)=\left(\begin{array}{l}
1 \\
0
\end{array}\right), \\
\mathbf{E}(90)=\left(\begin{array}{l}
0 \\
1
\end{array}\right) .
\end{gathered}
$$

Излучение с вертикальной поляризацией (2) направляется на неподвижный уголковый ретроотражатель 4 и служит опорным пучком. Излучение с горизонтальной поляризацией (1) попадает на подвижный уголковый ретроотражатель 5 , закрепленный, например, на катушке эталона килограмма, и оно является предметным пучком. При перемещении ретроотражателя 5 на величину $u(t)$ фаза предметного пучка относительно опорного изменится на величину

$$
\delta(t)=\frac{4 \pi}{\lambda} u(t),
$$

где $\lambda$ - длина волны стабилизированного лазерного излучения. 
С учетом этого вектор Джонса для предметного пучка надо переписать в виде

$$
\mathbf{E}(0)=\exp (i \delta)\left(\begin{array}{l}
1 \\
0
\end{array}\right) .
$$

Таким образом, на вход блока регистрации поступают два когерентных пучка лазерного излучения предметный (4) и опорный (2), имеющие ортогональную поляризацию и распространяющиеся по одному направлению. В блоке регистрации находятся следующие поляризационные элементы, которые описываются соответствующими матрицами Джонса [10].

1. Четвертьволновая пластинка 6, быстрая ось которой ориентирована под углом $45^{\circ}$ к оси $x$ :

$$
g w p(45)=\frac{1}{\sqrt{2}}\left(\begin{array}{cc}
1 & -i \\
-i & 1
\end{array}\right)
$$

2. Неполяризационный (обычный) светоделительный кубик 7 с соотношением 50\% на 50\% имеет следующие матрицы Джонса для отраженного (reflection) и прошедшего (transmission) света:

$$
\begin{gathered}
b s r=\frac{1}{\sqrt{2}}\left(\begin{array}{cc}
1 & 0 \\
0 & -1
\end{array}\right), \\
b s t=\frac{1}{\sqrt{2}}\left(\begin{array}{ll}
1 & 0 \\
0 & 1
\end{array}\right) .
\end{gathered}
$$

3. Для поляризационных светоделительных кубиков 3 и 10 матрица Джонса для прошедшего излучения совпадает с матрицей Джонса для линейного поляризатора, ориентированного под углом $0^{\circ}$ к оси $x$ :

$$
p b s_{x}=\left(\begin{array}{ll}
1 & 0 \\
0 & 1
\end{array}\right)
$$

а для излучения, отраженного от светоделительной грани - с матрицей Джонса для линейного поляризатора, ориентированного под углом $90^{\circ}$ к оси $x$, т.е. вдоль оси $y$ :

$$
p b s_{y}=\left(\begin{array}{cc}
0 & 0 \\
0 & -1
\end{array}\right) .
$$

4. Матрица Джонса для линейного поляризатора 8 , ориентированного под углом $45^{\circ}$ к оси $x$, равна

$$
p_{45}=\frac{1}{2}\left(\begin{array}{ll}
1 & 1 \\
1 & 1
\end{array}\right) \text {. }
$$

Если линейный поляризатор ориентирован под произвольным углом $\theta$ к оси $x$, то его матрица Джонса будет такая

$$
p(\theta)=\left(\begin{array}{cc}
\cos ^{2} \theta & \sin \theta \cos \theta \\
1 \sin \theta \cos \theta & 1 \sin ^{2} \theta
\end{array}\right) .
$$

Согласно $[10,11]$, преобразование поляризации рядом последовательно расположенных оптических устройств описывается результирующей матрицей, которая вычисляется как произведение соответствующих частных матриц, причем порядок перемножения матриц обратен порядку прохождения излучением системы оптических элементов.

В первом канале оба пучка (предметный и опорный) сначала проходят четвертьволновую пластинку 6 , далее отражаются от светоделительной грани обычного (неполяризационного) кубика 7 и, наконец, проходят через линейный поляризатор 8 , ориентированный под углом $45^{\circ}$ к оси $x$.

Обозначим результирующую матрицу для первого канала через K1, тогда ее можно записать в виде

$$
\mathbf{K 1}=q w p(45) b \operatorname{sr} p(45) .
$$

Согласно формализму Джонса, излучение, прошедшее через один или несколько последовательно расположенных поляризационных элементов, описывается произведением входного вектора Джонса на результирующую матрицу Джонса. Поэтому векторы Джонса для излучения, прошедшего первую систему поляризационных элементов, можно записать в виде

$$
\begin{aligned}
& \mathbf{E}_{1}(0)=\mathbf{K 1} \cdot \mathbf{E}(0)=\frac{\sqrt{2}}{4} \exp \left(i \frac{\pi}{4}\right) \exp (i \delta)\left(\begin{array}{l}
1 \\
1
\end{array}\right), \\
& \mathbf{E}_{1}(90)=\mathbf{K 1} \cdot \mathbf{E}(90)=\frac{\sqrt{2}}{4} \exp \left(i \frac{\pi}{4}\right) \exp (i \pi)\left(\begin{array}{l}
1 \\
1
\end{array}\right) .
\end{aligned}
$$

Таким образом, на первый фотодетектор через коллиматор 9 на рис. 1 приходит сумма когерентных световых полей, которые описываются коллинеарными векторами (13), (14), параллельными оси пропускания поляризатора 8 :

$$
\begin{aligned}
\mathbf{E}_{d 1}= & \mathbf{E}_{1}(0)+\mathbf{E}_{1}(90)=\frac{\sqrt{2}}{4}[\exp (i \pi) \\
& +\exp (i \delta)] \exp \left(i \frac{\pi}{4}\right)\left(\begin{array}{l}
1 \\
1
\end{array}\right) .
\end{aligned}
$$

Данная сумма полей направляется на первый фотодетектор, который регистрирует интенсивность излучения, описываемую произведением вектора Джонсона на комплексно сопряженный ему транспонированный вектор [10,11], поэтому

$$
\begin{aligned}
I_{d 1}(t) & =\frac{1}{4}\left|[\exp (i \pi)+\exp (i \delta)] \exp \left(i \frac{\pi}{4}\right)\right|^{2} \\
& =\frac{1}{2}\{1+\cos [\delta(t)-\pi]\} .
\end{aligned}
$$

Таким образом, в первом канале фотодетектор регистрирует гармонический сигнал (16), фаза которого 


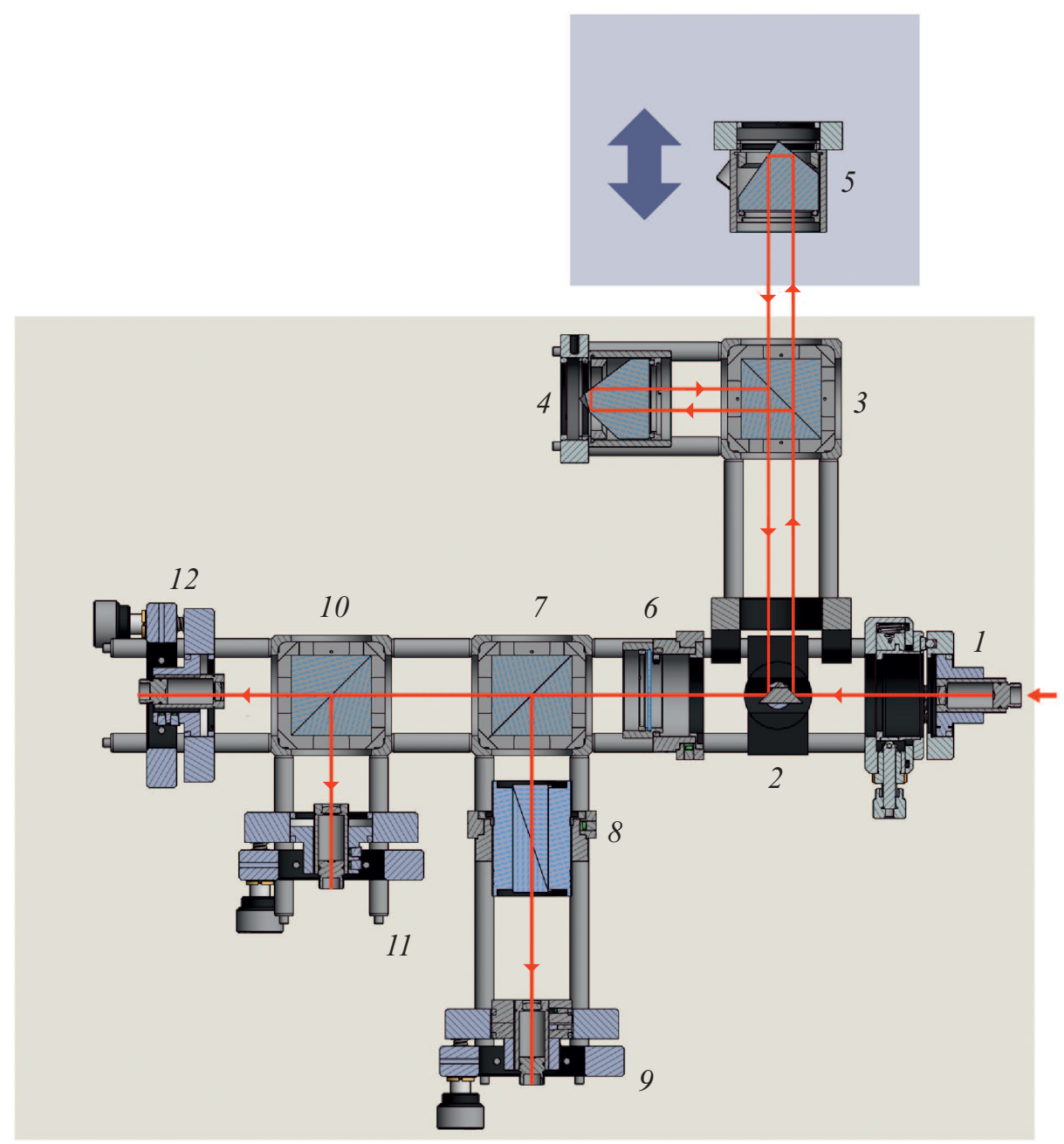

Рис. 1. Чертеж макета гомодинного квадратурного интерферометра перемещений: 1 - волоконный коллиматор для ввода лазерного излучения от стабилизированного по частоте Не-Nе-лазера (не указан); 2 - призма с зеркальными гранями; 3 поляризационный светоделительный кубик; 4 - неподвижный трехгранный ретроотражатель (триппель-призма); 5 - подвижный трехгранный ретроотражатель (триппель-призма); 6 - четвертьволновая пластинка, быстрая ось которой расположена под углом $45^{\circ}$ к горизонтальной плоскости; 7 - неполяризационный светоделительный кубик; 8 - линейный поляризатор под углом $45^{\circ}$ к горизонтальной плоскости; 9 - волоконный коллиматор, ведущий к первому фотодетектору; $10-$ поляризационный светоделительный кубик; 11 - волоконный коллиматор, ведущий ко второму фотодетектору; 12 - волоконный коллиматор, ведущий к третьему фотодетектору.

связана с перемещением катушки $u(t)$ по формуле (3) и сдвинута на $-\pi \mathrm{rad}$.

Во втором канале оба пучка также сначала проходят четвертьволновую пластинку 6 , затем через обычный (неполяризационный) кубик 7 и отражаются от светоделительной грани поляризационного светоделителя 10. Обозначим результирующую матрицу для второго канала через K2, тогда ее можно записать в виде

$$
\mathbf{K} \mathbf{2}=q w p(45) b s t p b s_{y} .
$$

Поле излучения, прошедшего через эти последовательно расположенные поляризационные элементы, можно записать в следующем виде:

$$
\begin{array}{r}
\mathbf{E}_{2}(0)=\mathbf{K} 2 \cdot \mathbf{E}(0)=\frac{1}{2} \exp \left(i \frac{\pi}{2}\right) \exp (i \delta)\left(\begin{array}{l}
0 \\
1
\end{array}\right), \\
\mathbf{E}_{2}(90)=\mathbf{K} \mathbf{2} \cdot \mathbf{E}(90)=\frac{1}{2} \exp (i \pi)\left(\begin{array}{l}
0 \\
1
\end{array}\right)
\end{array}
$$

Таким образом, на второй фотодетектор через коллиматор 11 на рис. 1 приходит сумма когерентных световых полей, которые описываются коллинеарными векторами (18), (19), лежащими в вертикальной плоско- 
сти:

$$
\begin{aligned}
& \mathbf{E}_{d 2}=\mathbf{E}_{2}(0)+\mathbf{E}_{2}(90) \\
& =\frac{1}{2}\left[\exp \left(i \frac{\pi}{2}\right)+\exp (i \delta)\right] \exp \left(i \frac{\pi}{2}\right)\left(\begin{array}{l}
0 \\
1
\end{array}\right) .
\end{aligned}
$$

Данная сумма полей направляется на второй фотодетектор, который регистрирует интенсивность:

$$
\begin{aligned}
I_{d 2}(t) & =\frac{1}{4}\left|\left[\exp \left(i \frac{\pi}{2}\right)+\exp (i \delta)\right] \exp \left(i \frac{\pi}{2}\right)\right|^{2} \\
& =\frac{1}{2}\left\{1+\cos \left[\delta(t)-\frac{\pi}{2}\right]\right\} .
\end{aligned}
$$

Видно, что во втором канале фотодетектор регистрирует также гармонический сигнал (21), фаза которого связана с перемещением катушки $u(t)$ по формуле (3) и сдвинута на $-\pi / 2$.

В третьем канале оба пучка также сначала проходят четвертьволновую пластинку 6 , затем через обычный (неполяризационный) кубик 7 и проходят через поляризационный светоделитель 10 на рис. 1. Обозначим результирующую матрицу для третьего канала через K3, тогда ее можно записать в виде

$$
\mathbf{K 3}=q w p(45) b s t p b s_{x} .
$$

Поле излучения, прошедшего через эти последовательно расположенные поляризационные элементы, можно записать в следующем виде:

$$
\begin{array}{r}
\mathbf{E}_{3}(0)=\mathbf{K} \mathbf{3} \cdot \mathbf{E}(0)=\frac{1}{2} \exp (i \delta)\left(\begin{array}{l}
1 \\
0
\end{array}\right), \\
\mathbf{E}_{3}(90)=\mathbf{K} \mathbf{3} \cdot \mathbf{E}(90)=\frac{1}{2} \exp \left(-i \frac{\pi}{2}\right)\left(\begin{array}{l}
1 \\
0
\end{array}\right) .
\end{array}
$$

Таким образом, на третий фотодетектор через коллиматор 12 на рис. 1 приходит сумма когерентных световых полей, которые описываются коллинеарными векторами $(23),(24)$, лежащими в горизонтальной плоскости:

$$
\mathbf{E}_{d 3}=\mathbf{E}_{3}(0)+\mathbf{E}_{3}(90)=\frac{1}{2}\left[\exp \left(-i \frac{\pi}{2}\right)+\exp (i \delta)\right]\left(\begin{array}{l}
1 \\
0
\end{array}\right) .
$$

Данная сумма пучков направляется на третий фотодетектор, который регистрирует интенсивность:

$$
\begin{aligned}
I_{d 3}(t) & =\frac{1}{4}\left|\left[\exp \left(-i \frac{\pi}{2}\right)+\exp (i \delta)\right]\right|^{2} \\
& =\frac{1}{2}\left\{1+\cos \left[\delta(t)+\frac{\pi}{2}\right]\right\} .
\end{aligned}
$$

В третьем канале фотодетектор регистрирует также гармонический сигнал (26), фаза которого связана с перемещением катушки $u(t)$ по формуле (3) и сдвинута на $+\pi / 2$.

Таким образом, показано, что приведенная последовательность поляризационных элементов позволяет получить три гармонических сигнала (16), (21) и (26), причем фаза двух сигналов (21) и (26) смещена относительно третьего сигнала (16) на $-\pi / 2$ и $+\pi / 2$, соответственно,

Из полученных трех сигналов можно сформировать два квадратурных сигнала без постоянной (аддитивной) составляющей. Вычитая сигналы друг из друга и используя тригонометрические формулы для суммы и разности углов, получим следующие выражения для квадратурных сигналов:

$$
\begin{aligned}
& I_{x}(t)=I_{d 2}(t)-I_{d 1}(t)=\sqrt{2} \sin \left[\delta(t)+\frac{\pi}{4}\right], \\
& I_{y}(t)=I_{d 3}(t)-I_{d 1}(t)=\sqrt{2} \cos \left[\delta(t)+\frac{\pi}{4}\right] .
\end{aligned}
$$

Поделив (27) на (28) и применив операцию $\operatorname{arctg}$, имеем

$$
\delta(t)=\operatorname{arctg}\left[\frac{I_{d 2}(t)-I_{d 1}(t)}{I_{d 3}(t)-I_{d 1}(t)}\right]-\frac{\pi}{4} .
$$

Используя выражение (3), получим окончательную формулу для вычисления искомого перемещения:

$$
u(t)=\frac{\lambda}{4 \pi} \operatorname{arctg}\left[\frac{I_{d 2}(t)-I_{d 1}(t)}{I_{d 3}(t)-I_{d 1}(t)}\right]-\frac{\lambda}{16} .
$$

После применения данной формулы необходимо выполнить операцию „сшивки“ фазы.

\section{Моделирование оптических полей, которые возникают после прохождения поляризационных элементов}

Как отмечается в работе [12], ключевой проблемой квадратурных интерферометров является чувствительность погрешности восстановления фазы к отклонению фазовых углов волновых пластинок (четверть- и полуволновых) от их номинального значения. Поэтому в настоящей работе на основе формализма матриц Джонса с помощью библиотеки символьных вычислений SymPy 1.6.2 в среде Python 3.8 проведен расчет оптических полей, которые возникают после прохождения поляризационных элементов, что позволило оценить влияние на измерения перемещений неточности их установки.

Для упрощения моделирования в оптической схеме интерферометра на рис. 1 использованы только два поляризационных элемента - четвертьволновая пластинка и поляризатор. Обычный светоделительный кубик исключен из рассмотрения, так как он не влияет на поляризационные характеристики излучения. Поляризационный 
светоделительный кубик на отраженное излучение действует как линейный поляризатор, ориентированный под углом $90^{\circ}$ к оси $x$, а на прошедшее излучение - как линейный поляризатор, ориентированный под углом $0^{\circ}$ к оси $x$. Поэтому при моделировании угол поворота поляризатора принимает три значения: 0,45 и 90.

Матрица оптической системы из этих двух оптических элементов представляет собой произведение матрицы Джонса поляризатора $p$ для произвольного угла поворота $\theta(11)$ и четвертьволновой пластины $q w p$ для произвольного угла $\varphi[10,11]$ :

$$
q w p(\varphi)=\frac{1}{\sqrt{2}}\left(\begin{array}{cc}
1+i \cos (\varphi) & i \sin (\varphi) \\
i \sin (\varphi) & 1-i \cos (\varphi)
\end{array}\right) .
$$

C помощью библиотеки символьных вычислений SymPy 1.6.2 были получены аналитические формулы для зависимости интенсивностей выходных сигналов $I_{1}, I_{2}, I_{3}$ от произвольного угла поворота четвертьволновой пластины $\varphi$, но для трех фиксированных значений угла $\theta$ поворота поляризатора: 0,45 и $90^{\circ}$.

Для получения точных квадратурных сигналов четвертьволновая пластина должна быть установлена под углом $45^{\circ}$. Для исследования влияния неточности установки этой пластины на погрешность измерений перемещений были дополнительно рассчитаны интенсивности выходных сигналов $I_{1}, I_{2}, I_{3}$ для углов, отличных от $45^{\circ}$, а именно для углов $46^{\circ}$ и $48^{\circ}$.

Для каждого набора полученных интенсивностей по формуле (30) восстанавливались временные зависимости перемещений $u_{1}(t), u_{2}(t)$ и $u_{3}(t)$ соответственно для углов $\varphi_{1}=46^{\circ}, \varphi_{2}=45^{\circ}$ и $\varphi_{3}=48^{\circ}$.

При равномерном движении уголкового ретроотражателя со скоростью $V$ для правильного угла поворота четвертьволновой пластинки $\varphi_{2}=45^{\circ}$ зависимость $u_{2}(t)$ представляет собой прямую, проходящую через начало координат под углом, тангенс которого равен скорости $V$.

Если четвертьволновая пластинка установлена под углом, отличным от $45^{\circ}$, то возникают отклонения от этого линейного закона, приводящие к погрешности измерения перемещений. Для более наглядной визуализации этих отклонений на рис. 2 приведены ,разностные“ зависимости $\left[u_{1}(t)-u_{2}(t)\right]$ и $\left[u_{3}(t)-u_{2}(t)\right]$, из которых видно, что для углов $\varphi$, отличающихся от точного угла на 1 и $3^{\circ}$, т.е. для углов 46 и $48^{\circ}$, эти отклонения не превышают $0.2 \mathrm{~nm}$.

\section{Моделирование квадратурных сигналов на фотоприемниках}

Для идеальной оптической схемы интерферометра квадратурные сигналы описываются выражениями (27) и (28). Однако реальные квадратурные сигналы отличаются от „идеальных“ из-за различного рода нелинейностей [4-8]. Они вызваны несовершенством поляризационных элементов, в первую очередь поляризационного

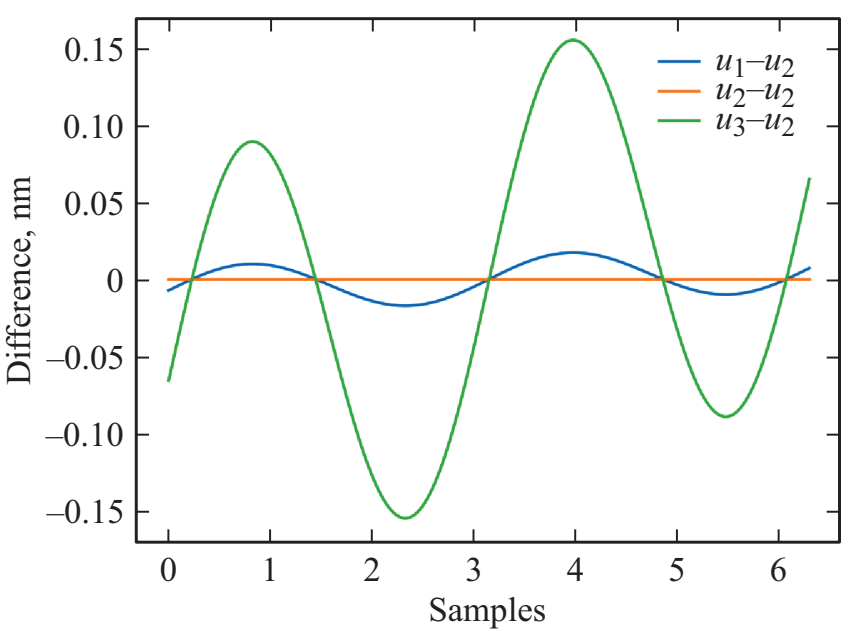

Рис. 2. Зависимость отклонений перемещений $\left(u_{1}-u_{2}\right)$ и $\left(u_{3}-u_{2}\right)$ от отсчетов времени.

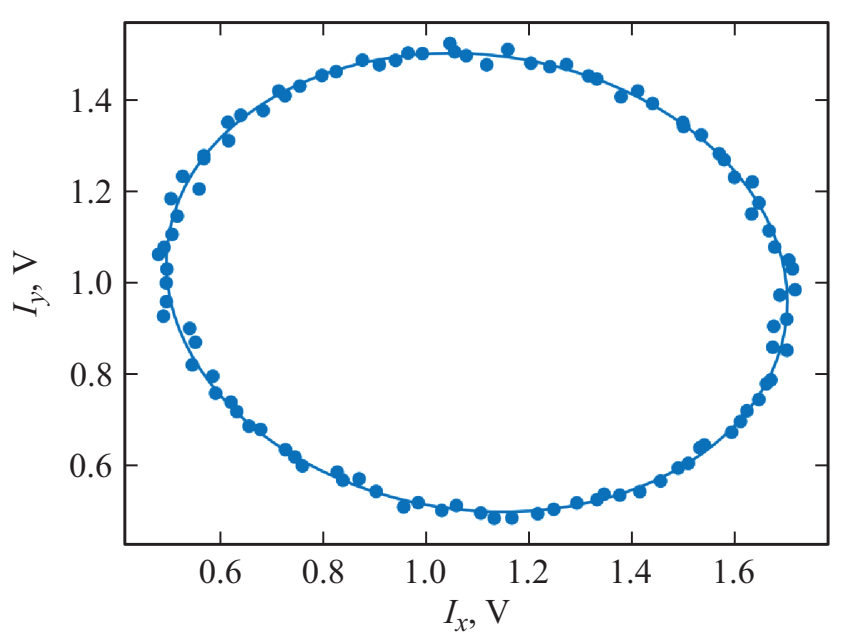

Рис. 3. Зависимость сигналов $I_{y}\left(I_{x}\right)$ при следующих параметpax сигналов: $a_{x}=1.0, b_{x}=0.5, a_{y}=1.1, b_{y}=0.6$ и $\Delta=5^{\circ}$ при уровне шума $R M S=0.01$.

светоделителя, различием в чувствительности и коэффициентах усиления детекторов [5]. Поэтому реальные квадратурные сигналы можно описать следующими выражениями:

$$
\begin{gathered}
I_{x}(t)=a_{x}+b_{x} \sin [\delta(t)], \\
I_{y}(t)=a_{y}+b_{y} \cos [\delta(t)+\Delta],
\end{gathered}
$$

где параметры $a_{x}, b_{x}$ описывают постоянный фон (аддитивная составляющая сигналов DC offsets); $a_{y}, b_{y}$ описывают амплитуду модуляции сигналов (мультипликативная составляющая сигналов $\mathrm{AC}$ ); параметр $\Delta-$ описывает наличие дополнительного фазового сдвига, кроме необходимого в $90^{\circ}$. Поэтому для реальных квадратурных сигналов пользоваться формулой (30) для определения перемещения $u(t)$ нельзя. 
Введем следующие обозначения:

$$
c_{x}=\frac{I_{x}(t)-a_{x}}{b_{x}}, \quad c_{y}=\frac{I_{y}(t)-a_{y}}{b_{y}} .
$$

Тогда из (32) и (33) несложно получить, что

$$
\delta(t)=\operatorname{arctg}\left(\frac{c_{x} \cos (\Delta)}{c_{y}+c_{x} \sin (\Delta)}\right) .
$$

Из (34) следует, что для определения фазы, кроме самих сигналов (32) и (33), необходимо также знать параметры сигналов $a_{x}, b_{x}, a_{y}, b_{y}$ и $\Delta$. Для их определения в работе [5] была предложена вычислительная процедура, названная эллиптическим фитингом. Если построить зависимость сигналов на фотоприемниках, задаваемых формулами (32), (33), друг от друга: $I_{y}\left(I_{x}\right)$, то полученная кривая будет представлять собой эллипс (фигуру Лиссажу). В реальном эксперименте квадратурные сигналы будут зашумлены, так как фотоприемники имеют определенный уровень шумов.

Моделирование проводилось по следующей схеме.

1. Сначала задавались параметры квадратурных сигналов, а именно число отсчетов сигнала за период $N$, параметры $a_{x}, b_{x}, a_{y}, b_{y}, \Delta$ и $R M S$ шума для точной фазы $\delta(t)$, изменяющейся по линейному закону при равномерном перемещении катушки.

2. Далее с помощью процедуры эллиптического фитинга находились параметры сигналов $a_{x}, b_{x}, a_{y}, b_{y}$ и $\Delta$.

3. По формуле (34) вычислялась новая фаза для реальных квадратурных сигналов.

4. Так как в формуле (34) присутствует arctg, то возможно наличие разрывов фазы, которые устранялись с помощью операции „сшивки“.

5. Вычисленная фаза аппроксимировалась прямой и из нее вычиталась исходная точная фаза.

6. Для перехода от фазы к перемещению умножаем полученную разность фаз на $\lambda / 4 \pi(\lambda=632.8 \mathrm{~nm})$ и

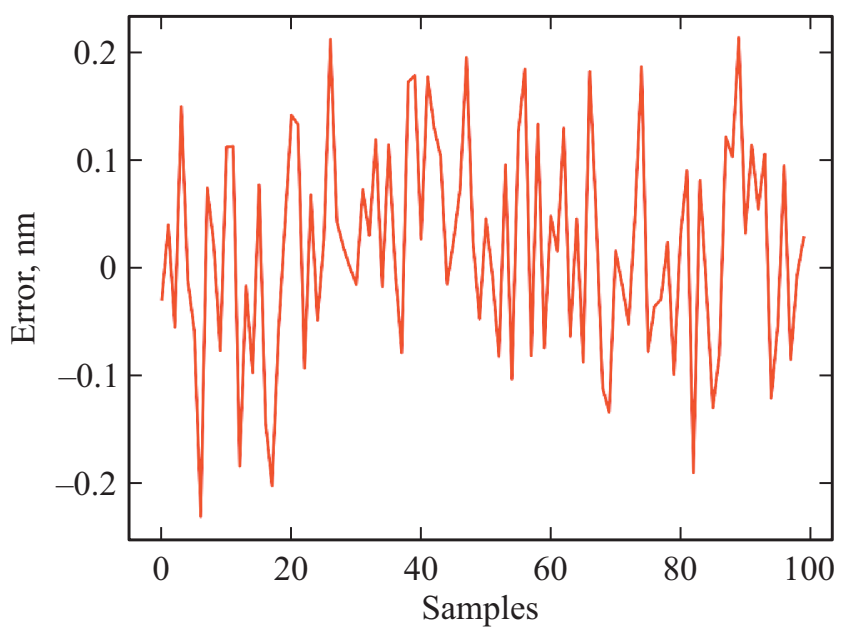

Pис. 4. Отклонение восстановленного перемещения от линейного после применения процедуры эллиптического фиттинга при уровне шума $R M S=0.001$. находим максимальное отклонение закона перемещения катушки от линейного.

В работе рассмотрена модель шума в виде аддитивной составляющей сигнала, имеющей гауссово распределение. Зависимость $I_{y}\left(I_{x}\right)$ при параметрах сигналов $a_{x}=1.0, b_{x}=0.5, a_{y}=1.1, b_{y}=0.6, \Delta=5^{\circ}$ и уровне шума $R M S=0.01$ представлена точками на рис. 3. На этом же рис. 3 сплошной линией показан эллипс, который был рассчитан с помощью процедуры эллиптического фиттинга, и он наилучшим образом аппроксимирует зависимость $I_{y}\left(I_{x}\right)$ в присутствии шума.

На рис. 4 приведен результат моделирования, проведенный по описанной схеме и для указанных выше параметров, но для меньшего уровня шума $R M S=0.001$, который соответствует реальному шуму фотоприемников в несколько $\mathrm{mV}$. Из графика видно, что погрешность восстановления искомого перемещения по реальным квадратурным сигналам амплитудой $1 \mathrm{~V}$ в присутствии шумов не превышает $0.3 \mathrm{~nm}$.

\section{Выводы}

На основе формализма матриц Джонса в работе получены формулы для квадратурных сигналов. На их основе проведено математическое моделирование оптических полей, которые возникают после прохождения поляризационных элементов. Показано, что неточность поворота четверьволновой пластинки в диапазоне от 1 до $3^{\circ}$ приводит к незначительной погрешности $0.2 \mathrm{~nm}$ в измерении перемещения. Также рассмотрен и промоделирован алгоритм компенсации нелинейных эффектов, возникающих при квадратичном детектировании интерференционных сигналов. Показано, что погрешность восстановления искомого перемещения по реальным квадратурным сигналам в присутствии шумов методом эллиптического фитинга не превышает $0.3 \mathrm{~nm}$.

В следующей работе предполагается описать экспериментальный образец гомодинного квадратурного интерферометра перемещений, привести результаты его опробования и метрологические характеристики.

\section{Финансирование работы}

Исследование выполнено в рамках Государственного контракта на выполнение НИР „Баланс“.

\section{Конфликт интересов}

Авторы заявляют, что у них нет конфликта интересов.

\section{Список литературы}

[1] Robinson I.A., Schlamminger S. // Metrologia. 2016. V. 53. N 5. P. A46.

[2] Коронкевич В.П., Соболев В.С., Дубнищев Ю.Н. Лазерная интерферометрия. Новосибирск: Наука, 1983. 212 с. 
[3] Watchi J. etal. Review of compact interferometers. [Электронный ресурс] Режим доступа: https://arxiv.org/pdf/1808.04175v2.pdf

[4] Wu C.-m., Su C.-s. // Meas. Sci. Technol. 1996. V. 7. N 1. P. 62.

[5] Wu C.-m., Su C.-s., Peng G.-S. // Meas. Sci. Technol. 1996. V. 7. N 4. P. 520.

[6] Cui J., He Z., Jiu Y., Tan J., Sun T. // Appl. Opt. 2016. V. 55. N 25. P. 7086.

[7] Ни P., Zhu J., Zhai X., Tan J.-B. // Opt. Express. 2015. V. 23. N 7. P. 8399.

[8] Yan L., Chen Z., Chen B., Xie J., Zhang S., Lou Y., Zhang E. // Opt. Express. 2018. V. 26. N 4. P. 4818.

[9] Kim D., Woo B.C., Lee K.C., Choi K.B., Kim J.A., Kim J.W., Kim J. // Metrologia. 2014. V. 51. N 2. P. S96.

[10] Gerrard A., Burch J.M. Introduction to Matrix Methods in Optics. Wiley-Interscience, 1975. Перевод: Джсеррард A., Бёрч Джс.М. Введение в матричную оптику. М.: Мир, 1978. $341 \mathrm{c}$.

[11] Ищенко Е.Ф., Соколов А.Л. Поляризационный анализ. М.: Знак, 1998. 208 c.

[12] Cui J., He Z., Tan J., Sun T. // Opt. Express. 2016. V. 24. N 20. P. 23505. 